\title{
Die Gemeinsame Deutsche Arbeitsschutzstrategie - ein Erfolg?
}

In der Gemeinsamen Deutschen Arbeitsschutzstrategie (GDA) bündeln die staatlichen Arbeitsschutzbehörden und die gesetzlichen Unfallversicherungen seit 2008 ihre Anstrengungen für einen besseren Arbeitsschutz. Die Bilanz der ersten Fünf-Jahres-Periode der GDA kann eine Reihe von Erfolgen ausweisen, zeigt aber auch Schwächen auf, wenn es um den Nachweis der Wirksamkeit von Maßnahmen auf der betrieblichen Ebene geht. Der Beitrag vermittelt Eindrücke über die Arbeitsweise der Strategie, fasst wichtige Ergebnisse der ersten GDA-Dachevaluation zusammen und gibt Anregungen zur Weiterentwicklung der Arbeitsschutzstrategie. ${ }^{1}$

LOTHAR LIßNER, CARSTEN BRÜCK, ANDREAS STAUTZ

\section{Die GDA als politische Strategie}

Mit der Gemeinsamen Deutschen Arbeitsschutzstrategie (GDA) setzte Deutschland eine zentrale Forderung der EUKommission um, die mit der Gemeinschaftsstrategie für Sicherheit und Gesundheit am Arbeitsplatz 2007 - 2012 den Mitgliedstaaten nahelegte, ihren Arbeits- und Gesundheitsschutz organisatorisch zu bündeln und strategisch auf die Verminderung spezifischer Belastungen zu fokussieren. Im deutschen Fall war auch der Bericht des Senior Labour Inspectors Committee (SLIC) von Bedeutung. Dieser Europäische Ausschuss Hoher Arbeitsschutzbeamter besuchte 2004 Deutschland und kritisierte Defizite in der Kooperation zwischen staatlichen Aufsichtsbehörden und Unfallversicherungsträgern sowie deren mangelnde strategische Ausrichtung. Nationale, kontrovers geführte Diskussionen über das duale Aufsichtssystem gaben zusätzliche Anstöße. So wurde von Teilen der Arbeitgeberverbände der Vorwurf übersteigerter Bürokratie und undurchschaubarer Regelungen geäußert. Gewerkschaften kritisierten die mangelnden Ressourcen der Aufsicht für die Prävention und die daraus resultierenden Defizite im Arbeitsschutz. Die GDA ist somit auch ein Ergebnis einer intensiven politischen und fachlichen Debatte über die Notwendigkeit der Neujustierung der Rollen und Funktionsbestimmungen der öffentlichrechtlichen Arbeitsschutzinstitutionen und einer kohärenten Ausgestaltung ihrer Vorgehensweisen.
Auf Beschluss der 83. Konferenz der Arbeits- und Sozialminister der Länder (ASMK) im November 2006 erarbeitete eine speziell eingesetzte Arbeitsgruppe grundlegende Dokumente für die Entwicklung gemeinsamer nationaler Arbeitsschutzziele und Handlungsfelder. Das 2007 vorgestellte „Fachkonzept und Arbeitsschutzziele 2008-2012“ bezog sich ausdrücklich auf die EU-Gemeinschaftsstrategie, berücksichtigte aber auch eine Reihe anderer Dokumente und Quellen. Ein abgestimmtes GDA-Konzept wurde ein Jahr später von der 84. ASMK bestätigt. Operative Arbeitsprogramme wurden strategisch auf Wirtschaftssektoren und Beschäftigtengruppen mit entsprechend hohen Arbeitsunfallzahlen und Krankenständen sowie Defiziten im Arbeits- und Gesundheitsschutz ausgerichtet.

Den Empfehlungen der EU-Strategie folgend findet eine regelmäßige Evaluation der Umsetzung der nationalen Arbeitsschutzstrategie statt. Entsprechend dem Arbeitsschutzgesetz umfasst dies „die Evaluierung der Arbeitsschutzziele, Handlungsfelder und Arbeitsprogramme mit geeigneten Kennziffern“. Die GDA wird auch hinsichtlich ihrer

\footnotetext{
Die Autoren führten aufseiten der Kooperationsstelle Hamburg IFE im Auftrag der Nationalen Arbeitsschutzkonferenz (NAK) die Dachevaluation der ersten Periode der GDA durch. Die Kooperationsstelle Hamburg IFE GmbH arbeitete dabei in einem Konsortium mit derTNS INFRATEST Sozialforschung $\mathrm{GmbH}$.
} 
Gesamtwirkung auf das Arbeitsschutzsystem und die Sicherheit und Gesundheit bei der Arbeit bewertet (Dachevaluation).

Der folgende Beitrag skizziert die mit dieser Evaluation einhergehenden methodischen Probleme am Beispiel mehrerer zentraler Ziele: der Trägerkooperation, der Verbesserung des betrieblichen Arbeitsschutzes, der avisierten Senkung von Arbeitsunfallzahlen und Muskel-Skeletterkrankungen sowie der Entwicklung von Präventionskultur und Gesundheitskompetenz. Erfolge sind vor allem im erstgenannten Arbeitsfeld festzustellen. Bei den anderen übergeordneten Arbeitsschutzzielen lassen sich Erfolge hingegen nur punktuell nachweisen. Die Effekte der operativen, auf die betriebliche Ebene einwirkenden Arbeitsprogramme wurden nicht von den Autoren der Dachevaluation, sondern von den Akteuren der GDA selbst evaluiert, daher sind die jeweiligen Ergebnisse aus methodischen Gründen nicht kompatibel. Ergebnisse der im Rahmen der Dachevaluation durchgeführten repräsentativen Betriebs- und Beschäftigtenbefragung identifizieren vor allem Betriebe mit bis zu 50 Beschäftigten als Problemzone und verweisen darauf, dass den dortigen Defiziten nur langfristig und mit verstärkter Aktivität begegnet werden kann.

\section{Struktur der GDA}

Das Unfallversicherungsmodernisierungsgesetz (UVMG) schuf 2008 die Rechtsgrundlagen für die GDA im Arbeitsschutzgesetz und im Sozialgesetzbuch VII. Bund, Länder und Unfallversicherungsträger verständigten sich auf die GDA als ein in Perioden unterteiltes, auf Dauer angelegtes politisches Programm zur Stärkung von Sicherheit und Gesundheit am Arbeitsplatz. Dieses Programm zielt darauf ab, das Niveau des Arbeitsschutzes in den Betrieben durch die Weiterentwicklung der öffentlichen Arbeitsschutzstrukturen einschließlich ihrer rechtlichen Bestandteile und Rahmenbedingungen zu optimieren. Im Fokus der GDA stehen dabei die Harmonisierung der Gesetzgebung und des Vorschriften- und Regelwerks, die Abstimmung der Institutionen untereinander und ihrer praktischen operativen Tätigkeiten sowie die Kooperation der Institutionen mit Dritten. Die Umsetzung arbeitsschutzrechtlicher Vorschriften in den Betrieben und die betriebliche Organisation des Arbeitsschutzes selbst sollen verbessert, Präventionskultur und Gesundheitskompetenz gestärkt werden.

Die GDA ist eine Kooperation juristisch und organisatorisch getrennter Institutionen, die übergreifende Gesamtziele vereinbaren. Sie wird durch die Nationale Arbeitsschutzkonferenz (NAK) politisch gesteuert und zentral koordiniert. Die NAK setzt sich aus jeweils drei stimmberechtigten Vertretern des Bundes, der Arbeitsschutzbehörden der Länder und der Spitzenverbände der gesetzlichen Unfallversicherung zusammen; je drei Vertreter der Spitzenorganisationen der Arbeitgeber und Arbeitnehmer neh- men mit beratender Stimme an den Treffen der NAK teil. Das jährlich als Fachkonferenz stattfindende Arbeitsschutzforum ist als ständige Einrichtung der GDA gesetzlich verankert und soll die NAK bei der Wahrnehmung ihrer Aufgaben beraten.

Die GDA verfügt über kein eigenes Budget; gemeinsame besondere Ausgaben wie die Kosten für die Geschäftsstelle der NAK, das Arbeitsschutzforum, Information und Kommunikation sowie Evaluation werden von den drei Trägern nach Beschluss in der NAK gemeinsam getragen. In der ersten Strategieperiode waren insgesamt ca. $10 \%$ der Ressourcen der Aufsicht (Beratung und Überwachung) für die GDA vorgesehen, dieser Wert schwankte jedoch von Träger zu Träger sehr stark. Eine grobe Schätzung der Kosten der öffentlichen deutschen Arbeitsschutzinfrastruktur auf Basis der Zahl der Aufsichtspersonen (ca. 5.300) plus unterstützendem Personal sowie Sachausgaben (insgesamt rund 500 Mio. $€)^{2}$ ergibt ein Volumen von ca. 50 Mio. € pro Jahr für die GDA, ganz überwiegend in Form des Einsatzes bereits vorhandener personeller Ressourcen. Diese Bereitstellung personeller Ressourcen für die GDA führte zu Spannungen, die mit der GDA verbundenen Aufgaben wurden nicht von allen Aufsichtspersonen begrüßt. So fand sich bei einer Online-Befragung der Aufsichtspersonen eine Reihe von Stimmen, die von einer Doppelbelastung in der Aufsichtstätigkeit berichteten und von einem Interessenkonflikt zwischen bisheriger Aufsichtstätigkeit und den Vorgaben durch die GDA sprachen. Auch in den Abschlussberichten der GDA-Arbeitsprogramme wurden Ressourcenprobleme angesprochen; u. a. wurde die durchschnittliche Zeit für eine Betriebsbesichtigung unterschätzt. Teilweise mussten die selbst gesteckten Ziele - etwa Anzahl der intervenierten Betriebe - angepasst werden oder es kam zum Zielkonflikt mit Routineaufgaben.

Für die Strategieperiode 2008-2012 definierte das sogenannte Terms of Reference-Papier (ToR-Papier) ${ }^{3}$ die wichtigsten Angaben über Zielebenen, Arbeitsschutzziele und die entsprechenden Teilziele sowie die Handlungsfelder (Übersicht 1), Indikatoren, Erhebungsinstrumente und Datenquellen. Die Träger der GDA vereinbarten in Abstimmung mit den Sozialpartnern drei übergeordnete Arbeitsschutzziele mit gemeinsamen Handlungsfeldern:

- Reduktion der Häufigkeit und Schwere von Arbeitsunfällen, - Verringerung von Häufigkeit und Schwere von MuskelSkelett-Belastungen und Erkrankungen und

- Reduktion der Häufigkeit und Schwere von Hauterkrankungen.

2 Es gibt keine spezifisch ausgewiesenen Daten der Kosten der Aufsicht für Deutschland. Für diese sehr überschlägige Schätzung wurden die Zahl des Aufsichtspersonals und die Bruttopersonalkostenwerte des öffentlichen Dienstes benutzt (Bundesministerium 2009).

3 Terms of Reference, (ToR-Papier), Stand vom 09.06.2010 


\section{Zuordnung der Arbeitsschutzziele zu den elf Arbeitsprogrammen}

\begin{tabular}{|c|c|c|c|}
\hline Arbeitsschutzziele & $\begin{array}{l}\text { Reduktion der Häufigkeit und } \\
\text { Schwere von Arbeitsunfällen }\end{array}$ & $\begin{array}{l}\text { Reduktion der Häufigkeit und } \\
\text { Schwere von Muskel-Skelett-Be- } \\
\text { lastungen und Erkrankungen }\end{array}$ & $\begin{array}{l}\text { Reduktion der Häufigkeit und } \\
\text { Schwere von Hauterkrankungen }\end{array}$ \\
\hline $\begin{array}{l}\text { Arbeitsprogramme Kategorie I } \\
\text { (verbindliche und bundesweite } \\
\text { Umsetzung/alle GDA-Träger) }\end{array}$ & $\begin{array}{l}\text { Bau } \\
\text { Zeitarbeit } \\
\text { Transport }\end{array}$ & $\begin{array}{l}\text { Pflege } \\
\text { Büro }\end{array}$ & Haut \\
\hline $\begin{array}{l}\text { Arbeitsprogramme Kategorie II } \\
\text { (regional begrenzte Umsetzung/ } \\
\text { Beteiligung der GDA-Träger fakultativ) }\end{array}$ & Schulen & $\begin{array}{c}\text { Feinmechanik } \\
\text { Ernährungsindustrie } \\
\text { Hotellerie } \\
\text { ÖPNV }\end{array}$ & \\
\hline
\end{tabular}

Quelle: Darstellung der Autoren.

Der Priorisierungsprozess für die Arbeitsschutzziele der ersten Strategieperiode kann als ein partizipativ-pragmatischer Diskussionsprozess unter Einbeziehung wissenschaftlicher Datenaufbereitung beschrieben werden, der stark sozialpolitisch geprägt war (Meffert 2008). Für den Auswahlprozess zur Bewertung von Arbeitsschutzzielen wurden zu Beginn der Strategieperiode fünf Kriterienbereiche genannt: empirisch-statistischer Kriterienbereich, Relevanz für Beschäftigte und Unternehmen, Relevanz für die Träger der GDA und allgemeine Kooperationen, gesellschaftliche Relevanz sowie Realisierbarkeit (Umsetzbarkeit, Steuerbarkeit) (Gemeinsame Deutsche Arbeitsschutzstrategie 2007).

Die Umsetzung der drei ausgewählten prioritären Arbeitsschutzziele erfolgte in erster Linie in sechs bundesweiten und fünf regional begrenzten Arbeitsprogrammen.

Die Optimierung der Zusammenarbeit der Träger im dualen System, insbesondere der Aufsicht von Ländern und Unfallversicherungsträgern, ist eines der wesentlichen Kernelemente der GDA. Im angenommenen Wirkungszusammenhang der Zielebenen wird darin eine Grundvoraussetzung für die Erreichung der Arbeitsschutzziele der GDA gesehen.

\section{Evaluation der GDA}

\subsection{Methodik der Evaluation}

In die GDA-Dachevaluation flossen die Ergebnisse von repräsentativen quantitativen Befragungen und qualitativen Erhebungen ebenso ein wie statistische Daten, die unabhängig von der GDA ermittelt wurden (Kontext-Informationen). Die zusammenfassende Bewertung der Ergebnisse der elf GDA-Arbeitsprogramme war ebenfalls Aufgabe der Dachevaluation, gleichwohl erfolgte die Eva- luierung der Arbeitsprogramme durch die Arbeitsprogrammleitungen selbst. Die von der NAK-Geschäftsstelle vorgelegten Berichte sowie die Veranstaltungsevaluationen des Arbeitsschutzforums wurden ebenfalls in die Dachevaluation integriert. Dieser Methodenmix sollte der Komplexität der GDA gerecht werden, Schwächen einzelner Erhebungsverfahren ausgleichen und die gestellten Fragen möglichst umfassend und fundiert beantworten (mixedmethods-approach). In diesem Zusammenhang wurden auch neue Erhebungsmethoden entwickelt, die sich für ein periodenübergreifendes Langzeit-Monitoring der GDA eignen.

Die einzelnen Arbeitsprogramme, in deren Rahmen die Interventionen der Strategie in den Betrieben durchgeführt wurden, waren durch weitgehende fachliche Autonomie geprägt. Die staatliche Aufsicht und die Unfallversicherungsträger konnten im Rahmen der allgemeinen Vorgaben jeweils über Instrumente und Vorgehen entscheiden, um eine optimale Nutzung der vorhandenen Ressourcen und Kenntnisse und damit eine bessere Zielerreichung zu ermöglichen. Dieser fachliche Vorteil war aus Perspektive der Dachevaluation bei der Zusammenfassung der Ergebnisse von Nachteil. Die Planung der Datenerhebung in den Betrieben, d. h. die Auswahl der Betriebe, das Vorgehen bei der Datenerhebung selbst, die gewählten Begrifflichkeiten, die Auswertung und Beurteilung blieben in der Hoheit der jeweiligen Akteure und waren nicht mit der Dachevaluation harmonisiert.

Im Rahmen der Dachevaluation wurden eigene Erhebungen durchgeführt und eine Fülle neuer Daten gewonnen, u. a. zum Stand der betrieblichen Arbeitsschutzorganisation, zur Existenz geeigneter Gefährdungsbeurteilungen, über Einschätzungen von Arbeitsbedingungen und Arbeitsschutzvorschriften, zu betrieblicher Präventionskultur und individueller Gesundheitskompetenz sowie zur Zusammenarbeit der Aufsichtsdienste. Eine Untersuchung vergleichbaren Umfangs und Detaillierungsgrades liegt bisher nicht vor. Hierzu gehören insbesondere: 
- Die Ergebnisse einer repräsentativen Betriebsbefragung (6.500 Betriebe) und einer repräsentativen Beschäftigtenbefragung (5.512 Beschäftigte). ${ }^{4}$

- Die Ergebnisse einer Online-Befragung des Aufsichtspersonals der Arbeitsschutzbehörden der Länder und der Unfallversicherungsträger (2.006 Aufsichtspersonen). ${ }^{5}$

- Die Ergebnisse von 57 qualitativen Interviews mit Arbeitsschutzexperten und Teilnehmern des Arbeitsschutzforums.

- Die Auswertungen der Evaluationsberichte der elf Arbeitsprogramme.

Einige zentrale Ergebnisse der GDA-Dachevaluation werden nachfolgend an vier Beispielen erläutert.

\subsection{Befunde}

\subsubsection{Neuordnung des Regelwerks und Optimierung der Zusammenarbeit der Träger}

Mit dem 2011 von den Trägern der GDA und den Sozialpartnern unterzeichneten Leitlinienpapier zur Neuordnung des Vorschriften- und Regelwerks im Arbeitsschutz wurde das Verhältnis von staatlichem Recht zum autonomen Recht der Unfallversicherungsträger neu definiert und die Verfahren beschrieben, die eine Abstimmung der beiden Rechtsbereiche gewährleisten sollen. Der Spielraum der autonomen Rechtsetzung durch die Unfallversicherungsträger wurde deutlich eingeschränkt. Diesem ersten Schritt zur „Herstellung eines verständlichen, überschaubaren und abgestimmten Vorschriften- und Regelwerks" sind aber bisher keine weiteren aus dem zitierten GDA-Ziel hergeleiteten Rechtsetzungsaktivitäten gefolgt.

Für die Teilziele „abgestimmter Vollzug“ und „Steuerung der Zusammenarbeit auf Länderebene" verpflichteten sich die staatlichen Arbeitsschutzbehörden und die Unfallversicherungsträger auf eine arbeitsteilige und aufeinander abgestimmte Beratung und Überwachung der Betriebe. Für die GDAArbeitsprogramme konnten diese Rahmenvereinbarungen mit gemeinsamen Umsetzungsvereinbarungen untersetzt werden, die das konkrete Vorgehen und den jeweiligen Ressourceneinsatz festlegten. Als zentrales Element konnte zusätzlich die gemeinsame Leitlinie für ein abgestimmtes Aufsichtshandeln, eine gleichwertige Umsetzung von Arbeitsschutzvorschriften sowie die Förderung eines Daten- und Informationsaustausches zwischen den Ländern und Unfallversicherungsträgern zu Betriebsbesichtigungen umgesetzt werden.

In praktisch allen Berichten der GDA-Arbeitsprogramme wird die Zusammenarbeit der Träger untereinander als positiv beschrieben. Es finden sich Begriffe wie etwa „vertrauensvoll und kooperativ“, „hohe Kooperationskultur“, „gute und konstruktive Arbeitsatmosphäre“, „neue Qualität des gemeinsamen Handelns“. Auch wenn die Arbeitsprogramme gele- gentlich über Zielkonflikte in der Abgrenzung mit Routinetätigkeiten, über Schwierigkeiten beim Datenaustausch oder über Ressourcenprobleme berichten, steht doch die Betonung der guten Zusammenarbeit und des Erfolges der Arbeit in den Programmen im Mittelpunkt der Berichterstattung.

Das Aufsichtspersonal der Präventivdienste äußerte sich an vielen Stellen kritischer. In der Online-Befragung des Aufsichtspersonals konnten auf die offene Frage nach „Hindernissen einer umfassenden Abstimmung in der Zusammenarbeit“ zwischen staatlichen Aufsichtsdiensten und Unfallversicherungsträgern 999 Nennungen von Aufsichtspersonen ausgewertet werden. Die einzelnen Aspekte wurden isoliert und zu Gruppen zusammengefasst. Das Aufsichtspersonal nannte an erster Stelle strukturelle Probleme als Hindernis der Zusammenarbeit (in 36,1 \% der Nennungen), an zweiter Stelle folgten Ressourcenprobleme (26,5\%) (Kooperationsstelle Hamburg IFE 2013). Die GDA wurde des Öfteren als weitere zusätzliche Aufgabe beschrieben, die andere bisherige Aufgaben in den Hintergrund drängte. Die Aufsichtspersonen waren damit konfrontiert, dass nicht alle konzipierten Instrumente der GDA zum geplanten Zeitpunkt zur Verfügung standen. Es gab praktische Probleme bei der Zusammenarbeit, so etwa beim IT-gestützten Datenund Informationsaustausch, bei dem es erst nach Ende der Strategieperiode 2013 gelang, in der Frage des Datenschutzes die notwendige Rechtssicherheit herzustellen.

Beim Aufsichtspersonal steht der Wunsch nach gegenseitiger Information über die Ergebnisse durchgeführter Betriebsbesichtigungen mit 35,1\% ganz oben auf der Wunschliste. Die unterschiedlichen Vorgaben und das unterschiedliche Aufgabenverständnis der Aufsichtsdienste von Ländern und Unfallversicherungsträgern werden mit gut $60 \%$ mehrheitlich als Hindernisse für eine umfassende Abstimmung in der Zusammenarbeit gesehen. Es wäre an geeigneter Stelle zu fragen, welche Konsequenzen die Träger der GDA aus den Ergebnissen der Befragung gezogen haben, um die geschilderten Schwierigkeiten zu überwinden und die Arbeitszufriedenheit ihrer Beschäftigten zu erhöhen. In der jetzt laufenden zweiten Periode der GDA ist eine erneute Befragung der Aufsichtspersonen vorgesehen (möglicherweise 2017). Spätestens dann wird sich zeigen, ob korrigierende Maßnahmen eingeleitet wurden und sich die Arbeitsbedingungen der Aufsichtspersonen verbessert haben.

\subsubsection{Der Arbeitsschutz auf der betrieblichen Ebene}

Die Ergebnisse der repräsentativen Betriebs- und Beschäftigtenbefragung im Rahmen der GDA-Dachevaluation und

4 Ausführlich zu den Befragungen siehe: Kooperationsstelle Hamburg IFE/TNS Infratest (2013)

5 Ausführlich zur Online-Befragung siehe: Kooperationsstelle Hamburg IFE (2013) 
die Abschlussberichte der GDA-Arbeitsprogramme konnten, wie schon erwähnt, aufgrund unterschiedlicher methodischer Vorgehensweisen kaum verknüpft werden. Auf Basis der so vorliegenden Daten untersuchte die Dachevaluation die Praxis des betrieblichen Arbeitsschutzes anhand der zuvor festgelegten Indikatoren. ${ }^{6}$ Besondere Aufmerksamkeit richtete sich dabei auf die betriebliche Arbeitsschutzorganisation und die Durchführung von Gefährdungsbeurteilungen. Das Ergebnis weist deutliche Defizite im Arbeitsschutz aus, insbesondere in kleineren Betrieben.

Es zeigte sich, dass insgesamt nur $59 \%$ aller Betriebe eine den gesetzlichen Anforderungen entsprechende sicherheitstechnische Betreuung aufweisen. Die Defizite konzentrieren sich stark auf Betriebe mit weniger als 50 Beschäftigten. Der Anteil der Betriebe, die die gesetzlich geforderte betriebsärztliche Betreuung in Anspruch nehmen, beträgt nur $40 \%$. Auch dieser Wert ist stark von der Situation in der kleinsten Betriebsgrößenklasse geprägt; dort lassen sich nur drei von zehn Betrieben von einem Betriebsarzt unterstützen. Die öffentliche Verwaltung, das Gesundheits- und Sozialwesen und der Investitions- und Gebrauchsgüterbereich haben führende Werte bei der sicherheitstechnischen Infrastruktur; der Dienstleistungsbereich für Unternehmen, der Einzelhandel und das Gastgewerbe haben in diesem Punkt den größten Nachholbedarf.

Insgesamt führen nur $51 \%$ aller Betriebe in Deutschland eine Gefährdungsbeurteilung durch. Insbesondere das schlechte Ergebnis bei den Kleinstbetrieben (bis zehn Beschäftigte) wirkt sich negativ auf das Gesamtergebnis aus. Grundsätzlich gilt: Je größer ein Betrieb ist, umso eher wird eine Gefährdungsbeurteilung durchgeführt. In der Betriebsbefragung gaben gut $90 \%$ der Betriebe mit über 50 Beschäftigten an, eine Gefährdungsbeurteilung durchzuführen. Branche, Größenklasse und Belastungssituation sind hierbei wichtige Einflussfaktoren. Das Vorkommen physischer Belastungen scheint die Durchführung von Gefährdungsbeurteilungen zu befördern, die psychischen Belastungen werden in den Gefährdungsbeurteilungen im Allgemeinen nur unzureichend erfasst. Die Bereiche der Kommunikations-, Finanz- und sonstigen Dienstleistungen sowie die Dienstleistungen überwiegend für Unternehmen weisen den geringsten Anteil an Betrieben mit Gefährdungsbeurteilungen auf. Den höchsten Anteil an Betrieben mit Gefährdungsbeurteilungen hat die Nahrungsmittelerzeugung, gefolgt von der öffentlichen Verwaltung, dem Produktionsgüter- sowie dem Investitions- und Gebrauchsgüterbereich.

Die Abschlussberichte der operativen GDA-Arbeitsprogramme auf Basis von Daten aus Betriebsinspektionen (Aufsicht) sowie Beratungstätigkeiten (UV-Träger) fallen im Hinblick auf die vorhandene Organisation des betrieblichen Arbeitsschutzes sowie die Durchführung von Gefährdungsbeurteilungen positiver aus als die der Betriebsbefragung. Dies ist jedoch insbesondere auf die Größe der dort betrachteten und intervenierten Betriebe zurückzuführen. In keinem Arbeitsprogramm gab es eine repräsentative Auswahl, es wurde auch nicht versucht, durch eine Gewichtung der Zahlen ein repräsentatives Bild in der Auswertung zu gewährleisten.

Die Berichte der GDA-Arbeitsprogramme legen einen engen Zusammenhang zwischen dem Stand der betrieblichen Arbeitsschutzorganisation und der Qualität der betrieblichen Prävention nahe: In Betrieben mit angemessener sicherheitstechnischer Infrastruktur und angemessener Gefährdungsbeurteilung werden deutlich weniger Verstöße gegen Arbeitsschutzvorschriften registriert. Daten aus wiederholten Betriebsbesichtigungen zeigen, dass die Interventionen der Aufsichtsdienste im Rahmen der GDA einen positiven Effekt auf die betrieblichen Arbeitsschutzanstrengungen hatten. Beispielsweise kann die positive Entwicklung bei der betrieblichen Gefährdungsbeurteilung in allen Arbeitsprogrammen, die eine Zweitbesichtigung vornahmen, als ein Indiz für die Wirksamkeit des Aufsichtshandelns gewertet werden. Auch im Hinblick auf die weitere betriebliche Arbeitsschutzorganisation - klare Delegation der Verantwortlichkeiten, betriebsärztliche und sicherheitstechnische Betreuung, Existenz eines Arbeitsschutzausschusses, Unterweisungen - konnten die GDA-Arbeitsprogramme, die eine Zweiterhebung durchführten, jeweils eine Verbesserung feststellen. Die Fortschritte scheinen hier jedoch geringer auszufallen als bei der Gefährdungsbeurteilung.

Die Ergebnisse der Betriebsbefragung zeigen eine überwiegend positive Beurteilung der Arbeitsweise der Aufsichtsdienste: Eine deutliche Mehrheit von jeweils rund zwei Dritteln der Betriebe bewertet die Zuständigkeiten als gut überschaubar (67\%), sieht ihre Betriebsabläufe durch die Aufsichtstätigkeiten nur wenig gestört (72\%) und attestiert den Aufsichtsdiensten eine kompetente Beratungsleistung (68\%).

\subsubsection{Arbeitsunfälle und Arbeitsunfähigkeitstage}

Die Reduktion der Häufigkeit und Schwere von Arbeitsunfällen war Schwerpunkt der präventiven Aktivitäten in vier branchenbezogenen GDA-Arbeitsprogrammen (Bau, Zeitarbeit, Transport, mit Einschränkungen: Schulen). Die Senkung der Arbeitsunfallzahlen galt als ein zentraler Indikator für die Wirksamkeit der GDA-Maßnahmen in diesem Bereich. Da in den Arbeitsprogrammen nur spezielle Branchen einbezogen und näher untersucht wurden, griffen wir

6 Indikatoren entsprechend derTerms of Reference der GDA waren: ,Anteil der Betriebe mit systematischer Gefährdungsbeurteilung', ,Anteil der Betriebe mit Arbeitsschutzmanagementsystem (AMS)/Betrieblicher Gesundheitsförderung (BGF)/Unternehmermodell', ,Anteil der Betriebe, in denen die Verantwortlichen die wesentlichen Anforderungen aus dem Regelwerk kennen und sie als angemessen erachten', ,Anteil der Betriebe, die die Aktivitäten der GDA-Träger als angemessen/transparent/koordiniert/ zweckmäßig bewerten: 
TABELLE 1

Meldepflichtige Arbeitsunfälle je 1.000 Vollarbeiter in der Bauwirtschaft, 2003-2012

Angaben in absoluten Zahlen

\begin{tabular}{|c|c|c|c|c|c|c|c|c|c|c|}
\hline Jahr & 2003 & 2004 & 2005 & 2006 & 2007 & 2008 & 2009 & 2010 & 2011 & 2012 \\
\hline $\begin{array}{l}\text { Meldepfl. AU / } 1000 \text { Vollarb. } \\
\text { Bauwirtschaft }\end{array}$ & 73,12 & 70,30 & 66,96 & 70,33 & 66,60 & 67,32 & 65,13 & 66,54 & 63,68 & 58,72 \\
\hline Durchschnitt & & & 69,46 & & & & & 64,28 & & \\
\hline
\end{tabular}

im Rahmen der Dachevaluation zur Abschätzung der Wirkungen der GDA auf das Arbeitsunfallgeschehen auch auf Routinestatistiken zurück.

In Deutschland hat sich die Gesamtzahl der meldepflichtigen Arbeitsunfälle je 1.000 Vollarbeiter $^{7}$ im Bereich der gewerblichen Berufsgenossenschaften in den letzten 20 Jahren in etwa halbiert, die Arbeitsunfallzahlen sinken im langjährigen Trend. Um mögliche Wirkungen von präventiven Maßnahmen zu isolieren und den Einfluss von kurzfristigen Kontextfaktoren (u. a. Konjunkturentwicklung) abzuschwächen, wurde für die Dachevaluation ein Verfahren gewählt, die Arbeitsunfallzahlen des Fünfjahreszeitraums der ersten GDA-Strategieperiode 2008 bis 2012 mit den Zahlen der Jahre 2003 bis 2007 zu vergleichen.

Im Zeitraum 2003 - 2007 ereigneten sich im Bereich der gewerblichen Berufsgenossenschaften durchschnittlich 27,95 meldepflichtige Arbeitsunfälle je 1.000 Vollarbeiter, im Zeitraum der ersten GDA-Periode von 2008 - 2012 sank dieser Wert um 7,4\% auf 25,88. Dieser Rückgang entspricht im Wesentlichen dem allgemeinen Trend der letzten Jahrzehnte, ist aber trotzdem bemerkenswert, da deutliche Rückgänge bei bereits niedrigerem Niveau immer schwieriger zu erreichen sind. Ein noch stärkerer Rückgang im Vergleich der beiden Fünfjahreszeiträume ist bei den neuen Arbeitsunfallrenten mit minus 14,9\% zu verzeichnen. Die tödlichen Arbeitsunfälle gingen sogar um 25,9\% zurück.

Da die Bauwirtschaft ein besonders unfallträchtiger Bereich ist und ein GDA-Arbeitsprogramm hier seinen Schwerpunkt hatte, wurde in der Dachevaluation für diese Branche eine Sonderauswertung vorgenommen. Im Fünfjahreszeitraum von 2003 bis 2007 ereigneten sich im Bereich der Bau-Berufsgenossenschaften ${ }^{8}$ durchschnittlich 69,46 meldepflichtige Arbeitsunfälle je 1.000 Vollarbeiter. 2008 - 2012 sank der Durchschnittswert um rund 7,5\% auf 64,28. Im Bereich der gewerblichen Berufsgenossenschaften ergab sich, wie oben dargestellt, im Periodenvergleich ein Rückgang um 7,4 \% (Tabelle 1).

Auffällig ist hierbei die Arbeitsunfallquote in der Bauwirtschaft für 2011, die trotz belebter Konjunktur (BIP 2011: $+3,0 \%$ ) unter der Quote des Rezessionsjahres 2009 (BIP $-5,1 \%$ ) liegt und mit den GDA-Aktivitäten in Verbindung gebracht werden kann. Aufgrund der Vielfalt der Faktoren, die auf das Arbeitsunfallgeschehen einwirken, lässt sich der Beitrag der GDA zum Rückgang der Arbeitsunfallzahlen jedoch nicht genau quantifizieren. Hier wären weitere Untersuchungen hilfreich, die sich intensiv mit der Wirkung von exogenen Faktoren auf das Arbeitsunfallgeschehen beschäftigen und versuchen, diese ins Verhältnis mit den Wirkungen präventiver Maßnahmen zu setzen.

Ein Effekt der sechs spezifischen GDA-Arbeitsprogramme (Pflege, Büro, Feinmechanik, Ernährungsindustrie, Hotellerie und öffentlicher Personennahverkehr) auf die Entwicklung der Arbeitsunfähigkeitstage, die auf Muskel-Skelett-Erkrankungen (MSE) zurückgehen, ist in den Daten der ersten Strategieperiode nicht erkennbar. Dies liegt vor allem an den vielfältigen Ursachen und der langen Latenzzeit der Erkrankungen, die unter dem Begriff MSE subsumiert werden, die eine Wirkungsmessung des Einflusses von GDA-Maßnahmen im Zeitraum einer Fünfjahresperiode unmöglich machen.

\subsubsection{Präventionskultur und Gesundheitskompetenz}

Neben der Reduktion der Arbeitsunfähigkeitstage nennt die GDA-Strategie 2008-2012 die Entwicklung einer Präventionskultur aufseiten der Betriebe und einer Gesundheitskompetenz aufseiten der Beschäftigten als Indikatoren, um die Verringerung von Häufigkeit und Schwere von MSE zu überprüfen. Der Begriff der Präventionskultur entstammt der EU-Gemeinschaftsstrategie für Sicherheit und Gesundheit

7 „Ein Vollarbeiter entspricht der durchschnittlich von einer vollbeschäftigten Person im produzierenden Gewerbe und Dienstleistungsbereich tatsächlich geleisteten - nicht der tariflichen - Arbeitsstundenzahl“ (DGUV, Geschäfts- und Rechnungsergebnisse, 2012, S. 12). 2012 war die jährliche Arbeitszeit mit 1.580 Stunden berechnet.

8 Die heutige Berufsgenossenschaft der Bauwirtschaft (BG Bau) entstand im Mai 2005 aus dem Zusammenschluss von sieben Bau-Berufsgenossenschaften und derTiefbauBerufsgenossenschaft. 
am Arbeitsplatz, wurde von der GDA-Arbeitsgruppe MuskelSkelett-Erkrankung aufgegriffen und um den Begriff der Gesundheitskompetenz ergänzt. Leider gab es zwischen den GDA-Akteuren kein gemeinsames Verständnis zur näheren Definition dieser Indikatoren und ihrer Operationalisierung, sodass die in den verschiedenen GDA-Arbeitsprogrammen erhobenen Daten nicht zusammengeführt werden konnten. Trotzdem versuchte die Dachevaluation, Kernaussagen aus den unterschiedlichen Ansätzen zu isolieren und mit den Ergebnissen der schon genannten repräsentativen Betriebsund Beschäftigtenbefragung zu verbinden.

Die Betriebs- und Beschäftigtenbefragung fragte u. a. nach betrieblichen Zielen zum Erhalt und zur Förderung der Gesundheit der Beschäftigten, der Behandlung von Fragen der Sicherheit und des Gesundheitsschutzes in Abteilungs- oder Arbeitsgruppenbesprechungen, der Einbeziehung der Mitarbeiter bei Gefährdungsbeurteilungen, dem Angebot an Maßnahmen der betrieblichen Gesundheitsförderung, dem Umgang mit Sicherheitsmängeln, der Etablierung betrieblicher Arbeitsschutzregelungen und der betrieblichen Kultur des Umgangs mit Verstößen dagegen. In der Beschäftigtenbefragung ging es speziell um die Einhaltung betrieblicher Sicherheits- und Gesundheitsschutzvorschriften, um die aktive Beteiligung an der Verbesserung der betrieblichen Sicherheit und des betrieblichen Gesundheitsschutzes, um die Nutzung betrieblicher Angebote der Gesunderhaltung (z. B. Betriebssport/Gesundheitszirkel), aber auch um die Ausübung sportlicher Aktivitäten, die Wahrnehmung medizinischer Vorsorgeuntersuchungen und um ausgewogene Ernährung.

Die in diesen Befragungen erhobenen Daten zeigen, dass sich in über $85 \%$ der Betriebe Mechanismen zur Prävention unmittelbarer Sicherheitsgefährdungen - also rechtlich vorgeschriebene Formen des Arbeitsschutzes - etablieren konnten. Es gibt klare Sicherheitsregeln, Sicherheitsmängel werden gemeldet und von den betrieblich Verantwortlichen zeitnah beseitigt. Allerdings zeigt das betriebliche Engagement zur langfristigen Gesunderhaltung noch größere Defizite, denn rund $30 \%$ der Beschäftigten bemängeln, dass ihr Betrieb nicht viel für eine Minimierung langfristiger Belastungen tut.

Nur eine Minderheit von rund einem Fünftel (21\%) der Betriebe hat eine Strategie zum Erhalt und zur Förderung der Gesundheit der Beschäftigten festgelegt. Dort, wo sie existiert, wird sie überwiegend (83 \%) in Form einzelner Maßnahmen zur betrieblichen Gesundheitsförderung (BGF) umgesetzt. Die häufigsten angebotenen Einzelmaßnahmen im Bereich BGF sind Gesundheitschecks (31\% der Betriebe), gefolgt von Mitarbeiterbefragungen (27\%) und Krankenstandanalysen (22\%). Betriebssport, Gesundheitszirkel oder Suchtpräventionsmaßnahmen gibt es nur in etwa jedem zehnten Betrieb. Erst Betriebe ab 250 Beschäftigten bieten mehrheitlich Einzelmaßnahmen der betrieblichen Gesundheitsförderung an. Da Großbetriebe überproportional viele Beschäftigte repräsentieren, ist das Bild bezogen auf die Anzahl der Beschäftigten, die von Maßnahmen der betrieblichen Gesundheitsförderung erfasst werden, positiver. Dennoch betreffen die Einzelmaßnahmen in keinem Fall eine Mehrheit der Mitarbeiterinnen und Mitarbeiter, wenn man eine beschäftigtenproportionale Auswertung zugrunde legt.

Die Ergebnisse der Beschäftigtenbefragung zur Gesundheitskompetenz zeigen, dass eine Basis für ein positives Gesundheitsverhalten bei den Beschäftigten vorhanden ist. Eine überwiegende Mehrheit der Beschäftigten hält sich meist an die betrieblichen Arbeitsschutzregeln, greift bei Fehlverhalten anderer ein und fühlt sich mitverantwortlich. Etwa die Hälfte der Beschäftigten macht eigene Vorschläge. Der Anteil derjenigen, die freiwillige betriebliche Präventionsangebote wahrnehmen oder sich im privaten Bereich gesundheitsgerecht verhalten, ist demgegenüber geringer. $32 \%$ verzichten zumeist auf sportliche Betätigungen, haben eine zumindest teilweise unausgewogene Ernährungsweise (21\%) oder nehmen nicht an medizinischen Vorsorgeuntersuchungen teil (27\%).

Betriebliche Präventionskultur und individuelle Gesundheitskompetenz erscheinen insgesamt steigerungsfähig. Die im Rahmen der ersten GDA-Strategieperiode durchgeführten Arbeitsprogramme konnten in einigen Aspekten Verbesserungen im betrieblichen Bereich bewirken. Das Verhalten im Privatleben kann aber allenfalls nur indirekt beeinflusst werden. Analysen der Beschäftigtenaussagen zeigten, dass es einen Zusammenhang zwischen der Qualität der betrieblichen Information zum Arbeits- und Gesundheitsschutz und der individuellen Gesundheitskompetenz gibt. Gute Information zum Gesundheitsschutz ist offenbar ein Erfolgsfaktor für den Aufbau einer Gesundheitskompetenz und für die Entstehung einer Präventionskultur.

\section{Grenzen der Evaluation, Bewertung und Ausblick}

Die Dachevaluation konnte sich nur in dem Rahmen bewegen, der von der GDA und den Terms of Reference vorgegebenen war. Somit hat die Evaluation eines gesellschaftlichen Teilsystems ihre Grenzen in einem notwendigerweise beschränktem Evaluationszweck und Evaluationsgegenstand. Solche Grenzen führen häufig zu Erklärungsproblemen. Sie ergeben sich dort, wo Aspekte des Teilsystems nicht Gegenstand der Evaluation sind und/oder als nicht hinterfragbare oder unveränderbare ,externe Faktoren' eingestuft werden. Ein Beispiel: Zur Überwindung der Probleme des Informationsaustausches und der mangelnden Harmonisierung zwischen den Ländern und den Unfallversicherungsträgern wäre ein möglicher Vorschlag, Föderalismus und Dualismus im ,Politiksektor Arbeitsschutz' aufzugeben oder abzumildern. Auch wenn dieses vielleicht Schwierigkeiten beim Informationsaustausch minimieren würde und damit sinnvoll und ressourcensparend wäre, liegt es politisch jenseits des 
Einflussbereichs der Akteure und auch außerhalb des Einflussbereichs der Evaluation und möglicher Empfehlungen.

Möglich wäre aber eine intensivere Beschäftigung mit den Trägern der GDA in der zweiten Strategieperiode 2013 bis 2018, so z. B. die Beschreibung „GDA-relevanter Infrastrukturen in den GDA-Trägern“. Der Fokus auf „Zusammenarbeit der Träger" ist unserer Ansicht nach nicht ausreichend, um einzelne Entwicklungen der GDA hinreichend evaluieren zu können. Es geht auch um mittel- und langfristige Aspekte der Trägerinfrastruktur (Ausbildung, Fortbildung, Qualifikation, Ressourcenentwicklung), die eine effektive Zusammenarbeit mit den anderen Trägern und die Wirkung des einzelnen Trägers im Rahmen der GDA erhöhen oder verringern. Dies ist nicht als Gesamtanalyse der Träger gedacht, es geht um die reale und mögliche Bedeutung und Funktion der GDA in den Strukturen der Träger. Aber auch dies müsste politisch gewollt sein.

Ein weiteres Beispiel ist die Analyse der Arbeitsunfallentwicklung. Die Unfallhäufigkeit wird nicht allein durch Prävention, sondern auch durch exogene Faktoren bestimmt. Den Erfolg der GDA in diesem Bereich nur an einem Sinken der Arbeitsunfallzahlen festzumachen, ist mithin fragwürdig. $\mathrm{Zu}$ den exogenen Faktoren gehören u. a. wirtschaftliche und strukturelle Entwicklungen, technologischer und medizinischer Fortschritt, Arbeitskräfteentwicklung und Personaleinsatz, der Wandel betrieblicher Managementkonzepte, veränderte politische und gesetzliche Schwerpunktsetzungen, aber auch administrative Veränderungen der Erfassung und Kategorisierung von Arbeitsunfällen. Die Beschreibung und Analyse dieser Faktoren wäre eine quantitative Überforderung im Rahmen der Dachevaluation gewesen. Sie wäre aber hilfreich für die Politikberatung, zur Ableitung von Empfehlungen und Handlungsvorschlägen für Gewerkschaften, Arbeitgeberverbände und Unfallversicherungsträger. Eine realistische Einschätzung der Wirksamkeit der Präventionsanstrengungen auf die Arbeitsunfallentwicklung kann zu einer Neubewertung von Schwerpunktsetzungen in der Arbeitsschutzpolitik und bei der Ausstattung der Arbeitsschutzinfrastruktur führen. Welche exogenen Faktoren sind bedeutsam, aber weitgehend ungeregelt? Wo stößt die bisherige Form der Prävention an ihre Grenzen, wo sind neue, innovative Konzepte gefragt?

Auch konnten Versäumnisse in der Datengewinnung über die Realität des Arbeitsschutzhandelns in den Unternehmen im Rahmen der GDA-Evaluation nur teilweise "geheilt"werden. Es wäre beispielsweise schon lange möglich, einen gesicherten nationalen Überblick über die Hauptmängel im betrieblichen Arbeitsschutz zu erhalten, indem - ergänzt durch Interviews mit dem Aufsichtspersonal - anonym und kontinuierlich ein Prozentsatz aller Unfallberichte, aller Besichtigungsberichte und seit Inkrafttreten des Arbeitsschutzgesetzes auch der Gefährdungsbeurteilungen ausgewertet worden wäre.

Zukünftig sollte auch darauf geachtet werden, dass das Vorgehen in den operativen Arbeitsprogrammen mit der Planung der Dachevaluation besser abgestimmt und Aus- wahl der Betriebe, Formen der Datenerhebung, Begrifflichkeiten und Evaluationsmethodik zwischen Arbeitsprogrammen und Dachevaluation harmonisiert werden. Zudem sollten vorwiegend Prozessindikatoren und in geringerem Umfang Erfolgsindikatoren zum Einsatz kommen. Prozessindikatoren dienen der Überprüfung der Aktivitäten zur Erreichung der gesteckten Handlungsziele, lassen Rückschlüsse auf das vorhandene Handlungspotenzial in den Betrieben zu und beschreiben Vorgehensweisen, die nach allgemeiner Überzeugung zu mehr Sicherheit und Gesundheitsschutz führen.

Die von der Arbeitsschutzstrategie beabsichtigten mittelund langfristigen Wirkungen auf gesamtgesellschaftlicher Ebene, wie etwa die dauerhafte Reduzierung der Arbeitsunfälle und arbeitsbedingten Erkrankungen, die Senkung der Arbeitsunfähigkeitstage und der damit verbundenen Kosten sowie die Steigerung der Wettbewerbsfähigkeit, sind für die erste Strategieperiode den GDA-Interventionen kausal nicht zuzuordnen. Ein Grund hierfür ist, dass praktisch alle Datenerhebungen im Rahmen der GDA auf neu entwickelten Instrumenten basierten. Diese Erhebungen sind in der Regel als Nullmessungen anzusehen, die zwar einen Ist-Zustand abbilden, aber zumeist keine Entwicklungslinien beschreiben können. Dies könnte sich in der nächsten Strategieperiode ändern, wenn erneut Erhebungen mit (ggf. modifizierten) Instrumenten vorgenommen werden, die dann als Vergleichsmessungen zur ersten Periode auswertbar sind. Aber auch hier ist darauf hinzuweisen, dass der Einfluss externer Faktoren in Abgrenzung zu den Aktivitäten der GDA auf die dann feststellbaren Veränderungen extrem schwierig zu beschreiben sein wird.

In der Gesamtschau bewerten wir (trotz der genannten Einschränkungen) die Entwicklung der GDA als einen erheblichen Fortschritt gegenüber der Situation vor der ersten Strategieperiode. Die GDA ist ein großer Schritt in Richtung eines abgestimmten gemeinsamen Handelns mit allen Vorteilen für die Effektivität der vorhandenen Infrastruktur. Insofern könnte die GDA in ihrer konkreten Vorgehensweise auch für andere, ähnlich konstituierte Politikfelder - bei noch zu erbringenden nachweisbaren positiven Wirkungen - eine anregende Vorbildfunktion haben.

Ein weiteres positives Ergebnis der GDA ist zweifellos der Zugewinn an Wissen. Die Fülle an detaillierten Informationen über die betriebliche Arbeitsschutzorganisation und -praxis in Deutschland, über das Aufsichtshandeln sowie über die Ideen der GDA-Träger und der potenziellen Kooperationspartner zur Lage und Zukunft von Sicherheit und Gesundheit bei der Arbeit in Deutschland ist beachtlich. Die Frage wird aber sein, wie mit diesem Zugewinn an Wissen umgegangen wird. Ob sich beispielsweise die staatlichen Aufsichtsdienste und die Unfallversicherungsträger der Kritik der eigenen Mitarbeiterinnen und Mitarbeiter annehmen, Anregungen aufgreifen und altes institutionelles Denken überwinden. Ob entsprechende Lehren aus dem Erfolg betrieblicher Beratungen und Interventionen gezogen und dem Arbeitsschutz die Ressourcen gegeben werden, die 
nötig sind, um die Gesundheit der Beschäftigten umfassend zu fördern und zu schützen, bleibt abzuwarten.

Es ist abzusehen, dass die Zukunft der öffentlichen Institutionen, die sich mit Sicherheit und Gesundheit am Arbeitsplatz befassen, immer stärker von ihrem kompetenten Umgang mit dem Thema Gesundheit abhängen wird. Die Gewährleistung von Sicherheit bleibt zwar nach wie vor ein essenzielles Tätigkeitsfeld, vor allem in den unfallträchtigen und gefahrgeneigten Branchen und Tätigkeiten. Jedoch wird die techno-legalistische Tradition der behördlichen Aufsichtstätigkeit mit immer komplexeren, facettenrei cheren Anforderungen jenseits des Sicherheitswissens konfrontiert, für die es bisher oft noch keine einfachen Messgrößen und Standards und in der Folge kaum eindeutige und justiziable Anforderungen an die Betriebe gibt, z. B. bei psychosozialen Belastungen. ${ }^{9}$ Die GDA könnte hier als Pilot- und Experimentierfeld in der zweiten Strategieperiode wertvolle Pionierarbeit leisten, z. B. durch die Entwicklung nachvollziehbarer und anerkannter Prozessindikatoren, die nützliche Erkenntnisse für Steuerungsentscheidungen liefern, prozessuales Lernen fördern und durch ein gutes Monitoring dazu beitragen, gesundheitliche Belastungen und Risiken in der Arbeitswelt zu reduzieren.

\section{LITERATUR}

Bruhn, A./Frick, K. (2011): Why it was so difficult to develop new methods to inspect work organization and psychosocial risks in Sweden, in: Safety Science, 49 (4), S. 575-581

Bundesministerium für Arbeit und Soziales/Bundesanstalt für Arbeitsschutz und Arbeitsmedizin (2009): Sicherheit und Gesundheit bei der Arbeit (SUGA) Unfallverhütungsbericht Arbeit, Dortmund/Berlin/Dresden, http://www.baua. de/de/Informationen-fuer-die-Praxis/Statistiken/Suga/Suga.html

Deutsche Gesetzliche Unfallversicherung (DGUV) (2007-2012): Geschäfts- und Rechnungsergebnisse der gewerblichen Berufsgenossenschaften und Unfallversicherungsträger der öffentlichen Hand, Berlin, http://publikationen.dguv. de/dguv/udt_dguv_main.aspx?QPX=TUIEPTEwMDImQ0IEPTEwMDI3Jk1NU1Y 9TU1TVjl=

Gemeinsame Deutsche Arbeitsschutzstrategie/GDA-Fachkonzept (2007):

Fachkonzept und Arbeitsschutzziele 2008 - 2012, Stand: 12. Dezember 2007 http://www.gda-portal.de/de/Ziele/Fachkonzept.html

Hauptverband der gewerblichen Berufsgenossenschaften (HVBG) (2005): Geschäfts- und Rechnungsergebnisse der gewerblichen Berufsgenossenschaften, Sankt Augustin, http://www.dguv.de/medien/inhalt/zahlen/documents/GUR2005.pdf

Kooperationsstelle Hamburg IFE (2013): Auswertung der Befragung des Aufsichtspersonals, Hamburg, http://www.gda-portal.de/de/pdf/Befragung-Aufsichtspersonal.pdf? _blob=publicationFile\&v $=2$

Kooperationsstelle Hamburg IFE/TNS Infratest (2013): Zwischenbericht zur Dachevaluation der Gemeinsamen Deutschen Arbeitsschutzstrategie, Nationale Arbeitsschutzkonferenz (Hrsg.), Berlin, http://www.gda-portal.de/de/pdf/ GDA-Dachevaluation_Zwischenbericht.pdf?__blob=publicationFile\&v=6 Kooperationsstelle Hamburg IFE/TNS Infratest (2014); Lißner, L./Brück, C./ Stautz, A./Riedmann, A./Strauß, A.: Abschlussbericht zur Dachevaluation der Gemeinsamen Deutschen Arbeitsschutzstrategie, Geschäftsstelle der Nationalen Arbeitsschutzkonferenz (Hrsg.), Berlin, http://www.gda-portal.de/de/Evaluation/Evaluation2008-12.html

Meffert, K. (2008): Gemeinsame Deutsche Arbeitsschutzstrategie (GDA) - Entwicklung von gemeinsamen Arbeitsschutzzielen und Handlungsfeldern, in: BPUVZ - Zeitschrift für betriebliche Prävention und Unfallversicherung, (2), S. 49-53, http://www.dguv.de/medien/inhalt/praevention/gemein_strat/ documents/A_II_04_08.pdf

Terms of Reference, (ToR-Papier) (2010): Gemeinsame Deutsche Arbeitsschutz strategie/Dachevaluation, Stand vom 09.06.2010, http://www.gda-portal.de/de/ pdf/TOR-Dachevaluation.pdf

\section{AUTOREN}

LOTHAR LIßNER, Dipl.-Soziologe, Geschäftsführer der Kooperationsstelle Hamburg IFE, nationale, europäische und internationale Projekte, Studien und Evaluationen zum Themengebiet Sicherheit und Gesundheit bei der Arbeit.

I.lissner@kooperationsstelle-hh.de

CARSTEN BRÜCK, Diplom-Jurist, MES, wissenschaftlicher Mitarbeiter der Kooperationsstelle Hamburg IFE, nationale, europäische und internationale Projekte, Studien und Evaluationen zum Themengebiet Sicherheit und Gesundheit bei der Arbeit.

c.brueck@kooperationsstelle-hh.de

ANDREAS STAUTZ, Dipl.-Volkswirt / Dipl.-Sozialökonom, Journalist und freier Mitarbeiter der Kooperationsstelle Hamburg IFE. Projekttätigkeit und Veröffentlichungen zum Thema Sicherheit und Gesundheit bei der Arbeit.

an.stautz@t-online.de 